\title{
Daphnia inhibits the emergence of spatial pattern in a simple consumer-resource system
}

\author{
Gustavo S. Betini, ${ }^{1,3}$ Tal Avgar, ${ }^{2}$ Kevin S. McCann, ${ }^{1}$ and John M. Fryxell ${ }^{1}$ \\ ${ }^{1}$ Department of Integrative Biology, University of Guelph, Guelph, Ontario N1G 2 W1 Canada \\ ${ }^{2}$ Department of Biological Sciences, University of Alberta, Edmonton, Alberta T6G 2 E9 Canada
}

\begin{abstract}
Spatial self-organization can occur in many ecosystems with important effects on food web dynamics and the maintenance of biodiversity. The consumer-resource interaction is known to generate spatial patterning, but only a few empirical studies have investigated the effect of the consumer on resource distribution. Here we report results from a large aquatic mesocosm experiment used to investigate the effect of the consumer Daphnia magna on the distribution of its resource, the green algae Chlorella vulgaris. We maintained large tanks with capacity for 26,000 L with either algae or both algae and Daphnia in different temperature conditions. We found that the presence of $D$. magna inhibited spatial structure in algal distribution that arose as a consequence of increasing temperature. We conjecture that this homogenization effect might be caused by a combination of high mobility combined with high rates of algal consumption by Daphnia. Our study emphasizes the importance of both local constraints on growth and behavioral responses in either promoting or suppressing spatial self-organization in natural populations.
\end{abstract}

Key words: aquatic mesocosmos; Chlorella vulgaris; freshwater systems; green algae; movement; predatorprey system; scale-dependent feedback; self-organization; spatial variation.

\section{INTRODUCTION}

Some biological systems exhibit regular spatial patterns that arise as a result of ecological interactions occurring at a small spatial scale (e.g., cells, individuals or species), even in the absence of an internal or external controller (Rietkerk and van de Koppel 2008). Wellstudied examples of such spontaneous emergence include the horizontal patchiness of phytoplankton in aquatic ecosystems (Levin and Segel 1976, Abraham 1998), the patchy stands of trees and shrubs in semiarid tropical environments (Belsky 1994, Klausmeier 1999) and clusters of young mussels on coastal mudflats (van de Koppel et al. 2005, Liu et al. 2013).

Consumer-resource interaction can contribute to such spatial structure (Levin and Segel 1976, van de Koppel et al. 2005). For example, spatially explicit predator-prey models can generate spatial pattern formation when predation causes local depletion of the prey but is unevenly spread across the ecosystem, leading to spatial heterogeneity in prey abundance (Hassell et al. 1991, de Roos et al. 1998, Gurney et al. 1998). This predator-induced patterning can occur even when the environment itself is heterogeneous, i.e., when prey patchiness is also caused

Manuscript received 12 July 2016; revised 6 November 2016; accepted 3 January 2017. Corresponding Editor: Shelley E. Arnott.

[3E-mail: gsbetini@gmail.com by abiotic factors (de Roos et al. 1998). Such spatial pattern formation is of considerable interest, because under some conditions it can help to suppress population fluctuations and thereby increase long-term persistence of predator-prey systems (Hassell et al. 1991, de Roos et al. 1998, van de Koppel et al. 2005).

On the other hand, it is just as plausible that intense predation could even out spatial disparities in prey abundance, provided that predators concentrate their efforts disproportionately in sites with locally abundant prey. Some consumers are able to track their resources, spending more time where resource abundance is high (Flaxman and Lou 2009). Under this scenario, patches with high resource abundance could be more heavily harvested by the consumer than low abundance patches, which could homogenize consumer distribution and inhibit the emergence of spatial self-organization (de Roos et al. 1998). Food web theory also predicts that mobile consumers with high attack rates, such as Daphnia, are expected to create spatial homogenization, which in turn leads to increased overall interaction strengths and destabilization of ecosystems (McCann et al. 2005). Despite widespread acceptance of the theoretical importance of spatial predator-prey interactions on species coexistence (Hassell 1984), the biodiversity of ecosystems (Solé and Bascompte 2012) and as a stabilizing force in complex food webs (McCann et al. 2005, Kondoh 2007, Mougi and Kondoh 2012), empirical tests of the role of 
consumers in either promoting or inhibiting spatial variation in prey abundance are uncommon.

Here we present evidence from a mesocosm experiment that consumer-resource interactions inhibit spatial pattern formation by the resource population. To test the effect of predation on the spatial distribution of the prey, we tracked consumer and resource density in space and time in six large tanks, each tank with capacity of $26,000 \mathrm{~L}$. The tanks were inoculated with either the nonmotile green algae Chlorella vulgaris (prey) or green algae and the freshwater water flea Daphnia magna (consumer). Variation in light and temperature within and among tanks created the opportunity for spatial structure in algal distribution, which we investigated in the tanks inoculated only with algae. In the tanks with algae and Daphnia, we expected strong interaction with its resource because Daphnia are well known for their ability to cause significant declines in algal abundance in natural systems (Brooks and Dodson 1965, Scheffer 2013), due to their high feeding efficiency and ability to track areas with high food abundance (Lampert et al. 2003, Lampert 2005).

\section{Methods}

The use of a mesocosm to investigate the effect of consumers on the spatial structure of the algal population is important because it represents a compromise between the realism, but intractability, of field studies and convenience, but unrealistic, small scale of microcosms that could mask the emergence of spatial structure (Resetarits and Bernardo 1998, Schindler 1998, Cadotte et al. 2005). Particularly, our system allows the study of pattern formation of the resource in the absence of the consumer, which is difficult to study in aquatic systems under field conditions.

Time series studies were conducted in the Guelph Limnotron facility, which consist of six double-walled stainless steel cylinders. Each tank measures $3.5 \mathrm{~m}$ in height and $3.15 \mathrm{~m}$ in diameter (Fig. 1a). A total of 18 different sampling ports allow samples to be taken vertically (six points; $50 \mathrm{~cm}$ apart from each other) and horizontally (three points; 25,50 , and $100 \mathrm{~cm}$ from the wall of each tank, Fig. 1a). Water temperature was controlled by one sensor in each tank positioned at $90 \mathrm{~cm}$ from the top of the water column (Fig. 1a). The sensors were connected to a cooling system controlling the water temperature by circulating glycol in the cooling jackets surrounding the tanks. A central system modulated the opening of the valves connected to the cooling unit in order to maintain preset water temperature in the tank. This setting allowed for a vertical gradient of water temperature similar to thermoclines commonly found in natural lakes in North America (Fig. 1b, Hondzo and Stefan 1993, Dobiesz and Lester 2009). Moreover, to create a greater range of temperatures, pairs of tanks, one with the non-motile green algae $C$. vulgaris and another with both algae and D. magna, were maintained at average temperature of $15^{\circ}, 20^{\circ}$, and $25^{\circ} \mathrm{C}$ (Fig. 1b). An independent lighting system $(1 \times 1000 \mathrm{~W}$ metal halide and $1 \times 1000 \mathrm{~W}$ high pressure sodium bulbs) suspended within each tank provided sufficient photosynthetically active radiation for phytoplankton growth. During the entire experiment, lights were kept on a $12 \mathrm{~h}: 12 \mathrm{~h}$ light:dark cycle and the water was not disturbed (i.e., there was no forced circulation in the tanks).

The tanks were filled with $26000 \mathrm{~L}$ of raw well water that was first passed through sediment pleated filters (5 and $0.2 \mu \mathrm{m}$ ) to remove small particles and irradiated with UV light to kill living organisms in the well water. The algae and Daphnia cultures used to populate each tank were reared on COMBO-ANIMATE medium (Kilham et al. 1998) kept at $20^{\circ} \mathrm{C}$ with $16 \mathrm{~h}: 8 \mathrm{~h}$ dark:light cycle. The D. magna population was derived from a clone from the Environmental Science Department, University of Guelph and fed with C. vulgaris prior to the experiment. The algae population used in the experiment was obtained from the Canadian Phycological Culture Centre (CPCC) at the University of Waterloo, Canada (strain \#90). Each tank was initially inoculated with $650 \mathrm{~mL}$ of algal solution (density $\sim 5 \times 10^{6}$ algae cells $/ \mathrm{mL}$ ). We added nutrients to each tank twice a week throughout the experiment with $69 \mathrm{ml}$ of a liquid plant fertilizer ( $12 \%$ nitrogen, $4 \%$ phosphorus, $8 \%$ potassium) that was first dissolved in $10 \mathrm{~L}$ of tank water and then dispensed at the top of the tank. Three weeks after algal inoculation, we also inoculated three tanks with $2 \mathrm{~L}$ of Daphnia culture (initial population size $\sim 250$ D. magna composed of $\sim 20$ adults and $\sim 230$ juveniles). Besides D. magna and C. vulgaris, ciliates and ostracods (always a small fraction of Daphnia) were occasionally found in the tanks throughout the experiment. In the $25^{\circ} \mathrm{C}$ treatment tanks, we occasionally observed green cells with different shapes at the end of the experiment, which could suggest the presence of other species of algae. All of these occurred infrequently and at barely detectable densities, and were hence unlikely to affect the experiment.

To quantify the spatiotemporal variation in both algae and Daphnia density, we sampled each of 18 different sampling ports from each tank twice a week over $91 \mathrm{~d}$ (26 sampling events per tank; Fig. 2a, b). In all tanks, temperature was measured and algae density was estimated from each of 18 samples. To estimate algae density we used a hand-held fluorometer cued to the concentration of chlorophyll a (AquaFluor Handheld Fluorometer; [Turner Designs, San Jose, California, USA] excitation $460 \pm 20 \mathrm{~nm}$; emission $>665 \mathrm{~nm}$ ). From each 18 ports, we first collected $0.5 \mathrm{~L}$ of water and then averaged three readings ( $2 \mathrm{~mL}$ of water for each reading). We transformed fluorometer readings into algal density (cell/mL) using a calibration curve obtained by counting number of cells in samples collected from the tanks $(n=89)$. Cells were counted under the microscope by using a $0.1-\mathrm{mm}$ 
a

Tank

Side view

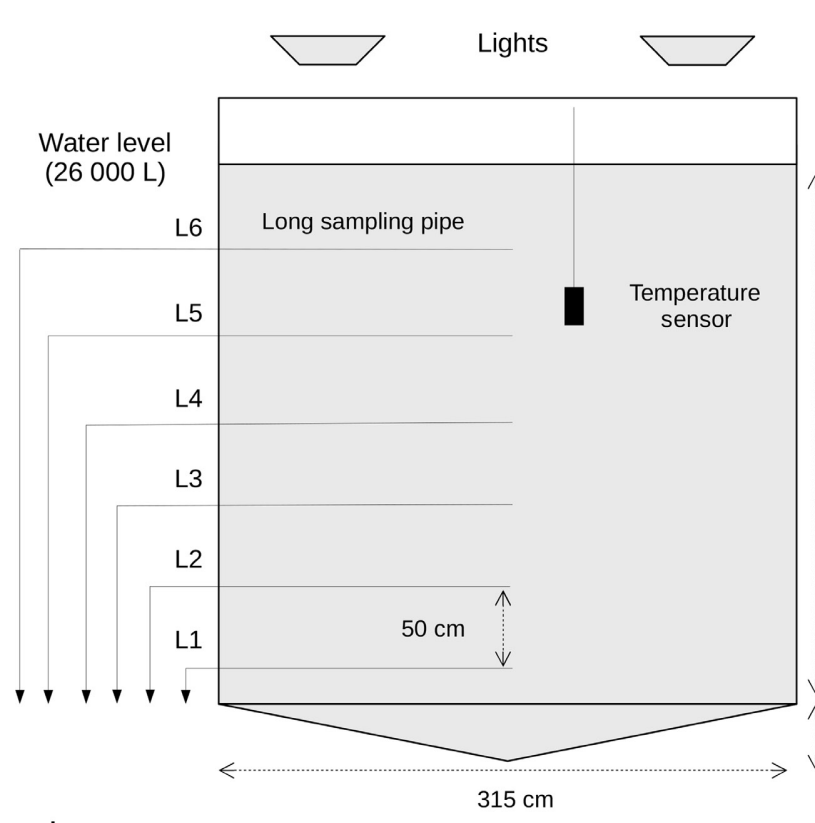

b

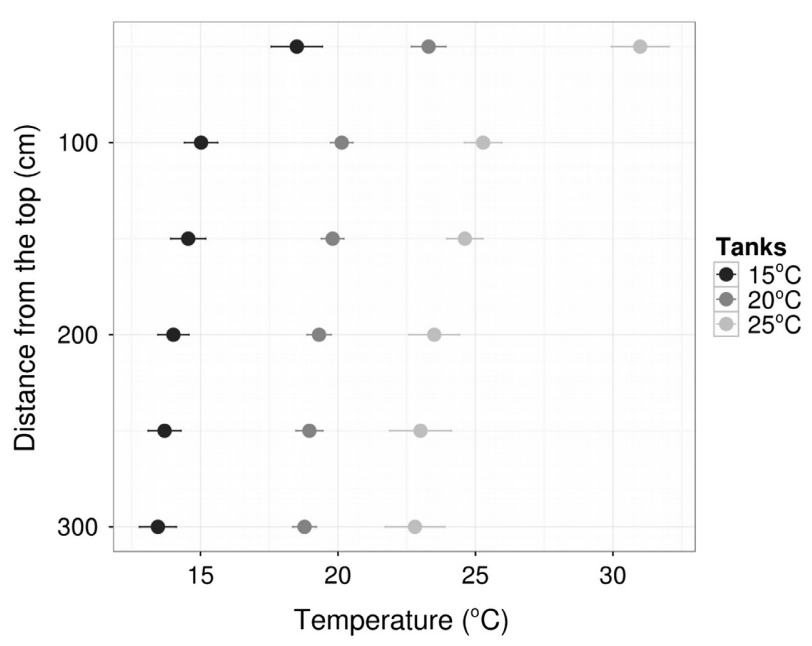

FIG. 1. (a) Schematic view of a limnotron tank. (b) Average temperature and standard deviation per sampling port over the period of the study for tanks with different preset temperature.

hemocytometer (calibration curve; number of cells $=31,170+7,332 *$ average fluorometer reading, $\left.R^{2}=0.82\right)$. In the tanks with Daphnia, three 1-L samples were taken from each sampling port, from which all individuals were counted and algae was measured as previously described. To obtain an estimate of Daphnia density (individuals per liter), we average the total counts of Daphnia in each liter, in each port. Water samples were returned to the tanks at the end of each sampling event. All samples were collected during the dark phase.

To quantify the degree of spatial structure in the tanks, we used an index of dispersion that, similarly to the coefficient of variation, measures whether a set of observations is clustered or dispersed with respect to a standard

$$
\sum_{i=1}^{n} \frac{\left(O_{i, t}-E_{t}\right)^{2}}{E_{t}}
$$

where $O_{i, t}$ is the algal density in port $i$ in tank $t$ in each sampling event and $E_{t}$ is the expected value in any port in tank $t$, calculated as the sum of algae density in each tank, in each sampling event, divided by the number of ports $(n=18)$. Thus, if algae were evenly distributed among all ports, the index of dispersion would be 0 . The higher the 

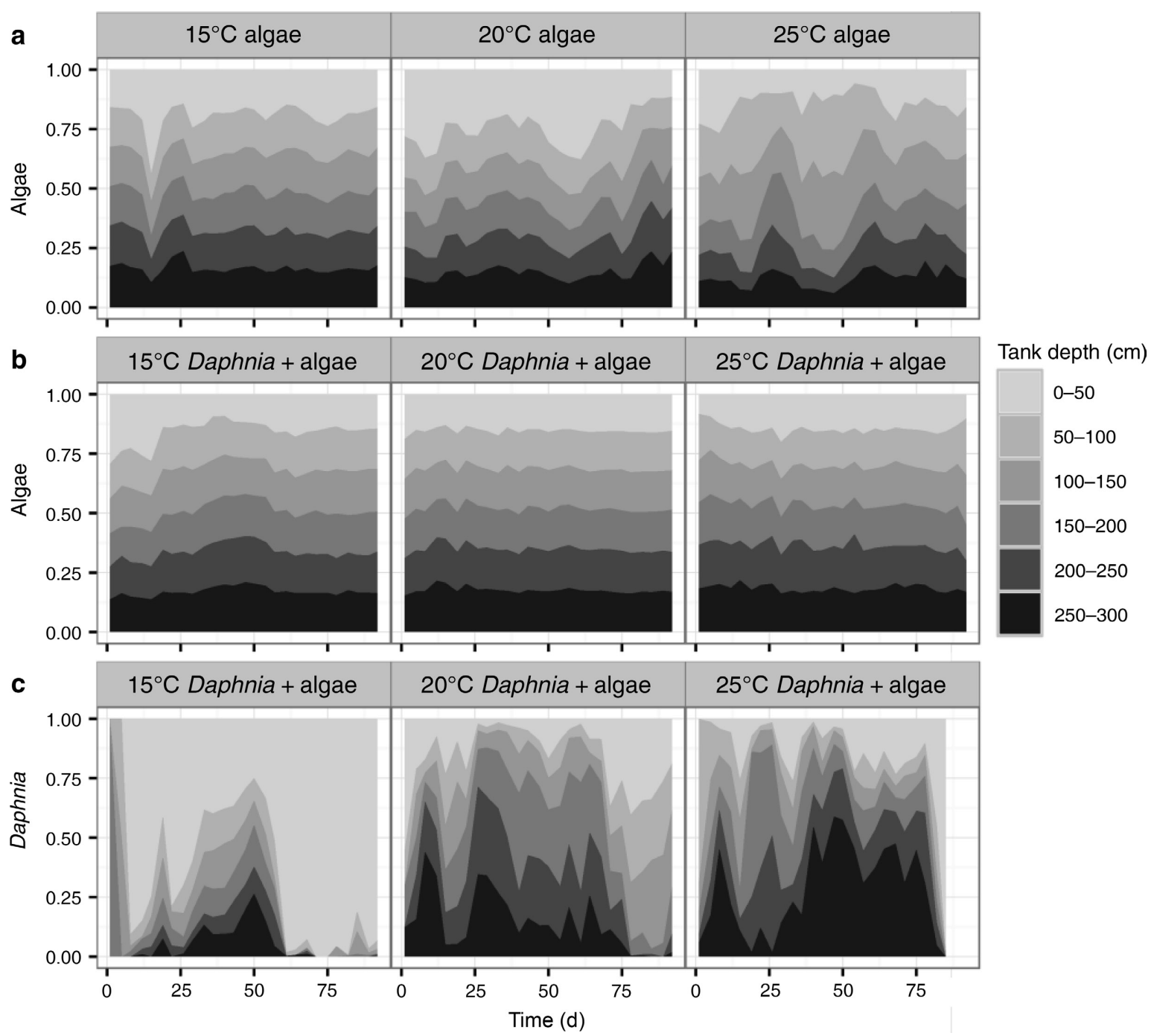

FIG. 2. Temporal variation in algal and Daphnia distribution in each tank. For simplicity, only the vertical structure is shown. Plots are the proportion of $(\mathrm{a}, \mathrm{b})$ algae and (c) Daphnia in each vertical port over the period of the study. Plots are the cumulative proportions in each vertical port (tank depth) of the total density of algae in tanks without (a) or with (b) Daphnia, and of the total density of Daphnia (c).

index, the stronger the patchiness in algal distribution within a single tank.

We investigated whether Daphnia promoted or inhibited spatial structure by comparing three linear mixed effect models (LMM) using the Akaike information criterion (AIC; Burnham et al. 2011). All models used the index of dispersion as a response variable (logtransformed to deal with heteroscedasticity) and tank identity as random effect. The first model was built under the assumption that algal distribution was only influenced by its own density and temperature. This model used average temperature and average algal density in each tank, in each sampling event as explanatory variables. To test whether the effect of the consumer on algal patchiness was similar regardless of the temperature, we added a third explanatory dummy variable "Daphnia" to identify weather the tanks had consumers or not. Finally, we tested whether the effect of Daphnia on algal distribution was temperature dependent through a third model that added an interaction term between temperature and Daphnia as an explanatory variable. To account for potential temporal autocorrelation in the residuals within each tank, we included in the model fit an auto-regressive term of order 1 using the corAR 1 class in function lme, which assumes that each residual value is a stochastic linear function of the previous residual value (thus accounting for temporal autocorrelation; Pinheiro and Bates 2009). For AIC comparison, models were fit by maximum likelihood estimation, but parameters were obtained with the restricted maximum likelihood method 
(Zuur et al. 2009). The code in $\mathrm{R}$ with all variables tested, plus the correlation structure was lme(index of dispersion $\sim$ average algal density + average temperature in each port $\times$ treatment, data $=$ data, random $=\sim 1 \mid$ tank, correlation $=\operatorname{corAR} 1($ form $=\sim 1 \mid$ tank $)$ ). For all analysis, we used data that were collected after the first individual of Daphnia was observed in the tanks ( 20 d after inoculation) and matched the same time period for the tanks without Daphnia.
To investigate the long-term effect of Daphnia on algae patchiness, we used a similar approach to compare spatial structure for the tanks at $25^{\circ} \mathrm{C}$ with and without Daphnia, which were sampled for $288 \mathrm{~d}$. Because we had only two tanks at $25^{\circ} \mathrm{C}$, we used generalized linear models (GLS) instead of LMMs, with the same first-order autocorrelation structure for the residuals. All analysis were performed in R ( $\mathrm{R}$ Core Team 2015). We used the package nlme to perform the LMMs and GLSs (Pinheiro et al.
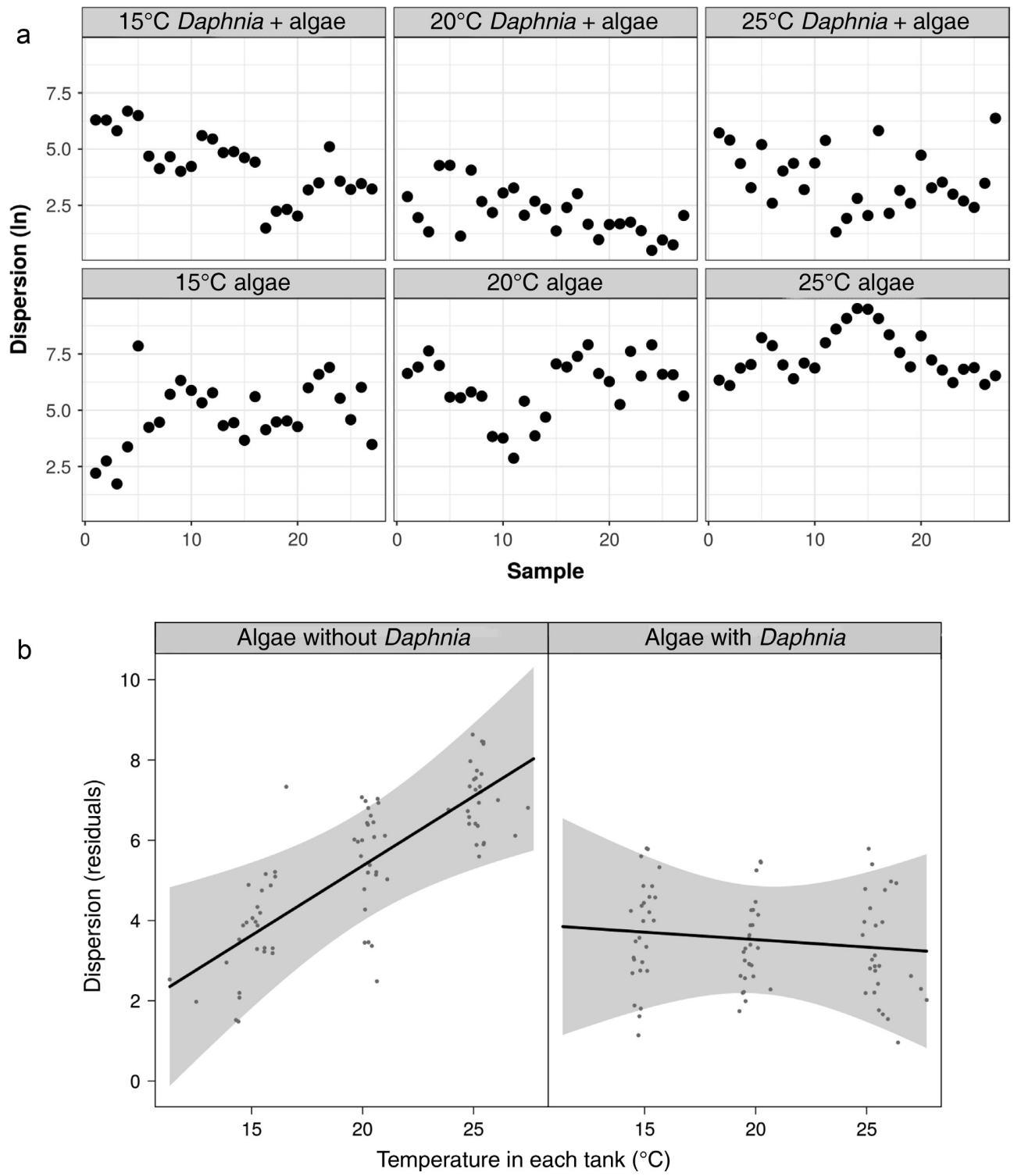

FIG. 3. (a) Temporal variation in the index of dispersion (a proxy for algae patchiness) and (b) conditional plots illustrating the effect of Daphnia magna and preset temperature on algal patchiness. Conditional plots were calculated from a linear mixed effect model with the index of dispersion as a response variable, tank ID as the random effect, and the interaction between mean tank temperature and treatment as explanatory variables. Mean algal density in each tank was entered as a covariate. Dots represent partial residuals, horizontal lines are prediction lines, and gray shaded areas are confidence intervals based on the model. 
TABLE 1. Akaike's information criterion (AIC) model selection parameters for competing models used to explain variation in algal distribution.

\begin{tabular}{|c|c|c|c|c|c|}
\hline Model & $\mathrm{df}$ & LogLik & $\mathrm{AIC}_{\mathrm{c}}$ & $\Delta \mathrm{AIC}$ & $w$ \\
\hline \multicolumn{6}{|l|}{ All tanks } \\
\hline 1. Algae + Temperature & 6 & -247.94 & 508.4 & 5.78 & 0.05 \\
\hline 2. Algae + Temperature + Daphnia & 7 & -245.63 & 506.0 & 3.35 & 0.15 \\
\hline 3. Algae + Temperature $\times$ Daphnia & 8 & -242.85 & 502.7 & 0 & 0.80 \\
\hline \multicolumn{6}{|l|}{ Tanks at $25^{\circ} \mathrm{C}$} \\
\hline 1. Algae & 4 & -253.71 & 515.7 & 6.42 & 0.04 \\
\hline 2. Algae + Daphnia & 5 & -249.44 & 509.3 & 0 & 0.96 \\
\hline
\end{tabular}

Notes: Temperature and Algae refer to mean temperature and mean algal density in each port, in each tank, respectively. Daphnia is a dummy variable to identify whether the tanks had Daphnia or not. Analysis with all tanks were run for a shorter period of time compared to analysis for the two tanks at $25^{\circ} \mathrm{C}$ (see Methods for details). Abbreviations: LogLik, log-likelihood; AIC $c$, Akaike's information criterion corrected for small sample size; $\triangle \mathrm{AIC}$, difference for model relative to the smallest AIC $c$ in the model set; $w$, Akaike weight, which is the approximate probability in favor of the given model from the set of models considered.

2016) and the package visreg to create the conditional plots (Breheny and Burchett 2013).

\section{RESULTS AND Discussion}

The most parsimonious model to explain variation in the index of dispersion incorporated mean algal density and the interaction between average temperature and whether the tank had Daphnia or not as explanatory variables. According to this model, the index of dispersion increased with temperature after controlling for the effect of algal density in each tank (Figs. 2 and 3a, b). This effect was significant only in the tanks without Daphnia (Table 1, Figs. 2a and 3a, b). There was no significant difference in the index of dispersion among the tanks with both algae and Daphnia (Fig. 3a, b; Table 2), suggesting that consumers inhibited spatial structure of the algae population. We found similar results when the analysis was done over a longer period of time (i.e., with the tanks with preset temperature at $25^{\circ} \mathrm{C}$; Table 1). The index of dispersion was lower over $288 \mathrm{~d}$ in the tank with Daphnia than in the tank with only algae (Table 2).

Many theoretical and a few empirical studies have highlighted the importance of consumer-resource interactions in the formation of spatial self-organized patterns (Levin and Segel 1976, Hassell et al. 1991, Rietkerk and van de Koppel 2008, Solé and Bascompte 2012). As we have shown here, however, consumers can also homogenize the spatial distribution of their food resources, even when spatial structure is caused by variation in abiotic conditions. We conjecture that this effect depends on the consumer's capacity to adjust its space-use behavior and demographic rates to local conditions (McCann et al. 2005, Silliman et al. 2013). Thus, predicting whether consumer-resource systems can create spatial self-organized patterns requires knowledge of the behavior of both consumer and its resource.

The patchiness in algal distribution observed in the tanks with only algae increased with temperature, suggesting that heterogeneity in abiotic conditions was responsible for the emergence of the spatial structure. This is consistent with studies showing that growth conditions for different strains of $C$. vulgaris is maximized at temperatures varying between $20^{\circ}$ and $25^{\circ} \mathrm{C}$ (Dauta et al. 1990), including the strain used here (Jarvis et al. 2016). This range of temperature was only achieved at the top of tank kept at $20^{\circ} \mathrm{C}$ and in the tank kept at $25^{\circ} \mathrm{C}$, which explains why algal distribution was more clustered in these tanks and more evenly distributed in the colder tank (i.e., $15^{\circ} \mathrm{C}$ ).

We suspect that the homogenization of algal distribution by Daphnia was enhanced by the pronounced mobility of the consumer combined with its high feeding efficiency. Pattern formation depends critically on the relative mobility of consumers relative to their resources. The typical mechanism behind pattern formation in

TABLE 2. Approximate $95 \%$ confidence intervals for the parameters in the most supported models according to AIC comparison.

\begin{tabular}{lccc}
\hline \hline Model & Lower & Estimate & Upper \\
\hline All tanks & & & \\
1. Intercept & -6.98 & -2.01 & 2.95 \\
2. Algae & 3.41 & $6.67 \times 10^{-6}$ & $9.92 \times 10^{-6}$ \\
3. Temperature & 0.11 & 0.35 & 0.58 \\
4. Daphnia & -4.65 & 5.83 & 16.31 \\
5. Daphnia $\times$ & -0.74 & -0.38 & -0.024 \\
$\quad$ Temperature & & & \\
Tanks at 25 ${ }^{\circ} \mathrm{C}$ & & & \\
1. Intercept & 3.68 & 4.62 & 5.57 \\
2. Algae & $7.27 \times 10^{-6}$ & $1.14 \times 10^{-5}$ & $1.16 \times 10^{-5}$ \\
3. Daphnia & -2.57 & -1.57 & -0.58 \\
\hline
\end{tabular}

Notes: Temperature and Algae refer to mean temperature and mean algal density in each port, in each tank, respectively. Daphnia is a dummy variable to identify whether the tanks had Daphnia or not. Analysis with all tanks were run for a shorter period of time compared to analysis for the two tanks at $25^{\circ} \mathrm{C}$ (see Methods for details). 
most spatially explicit models of consumer-resource interaction is a strong local demographic interactions between the consumer and the prey, combined with limited capacity for consumer redistribution via diffusive motion (Rietkerk and van de Koppel 2008, Solé and Bascompte 2012). Patterns arise because of local depletion of prey, creating prey heterogeneity that eventually contributes to collapse of the consumer population. Slow rates of discovery of regenerating prey patches by consumers in turn creates an opportunity for the prey population to restore its abundance without local consumer control. Daphnia is a highly efficient consumer (Lampert et al. 1986) and bench-top and field experiments have demonstrated, similarly to our experiments, that Daphnia can inhibit the growth of algae even at low levels of algal abundance (McCauley and Murdoch 1987, McCauley et al. 1999, Scheffer 2013). Moreover, Daphnia individuals from the same clonal population had swimming speeds varying between 0.3 and $0.8 \mathrm{~cm} / \mathrm{s}$ (Betini et al. 2016), which is consistent with swimming speeds reported elsewhere (Dodson et al. 1997, O'Keefe et al. 1998). This means that Daphnia individuals swimming in a straight line at maximum velocity could, in principle, cross the entire tank $(\sim 3 \mathrm{~m})$ in $\sim 10 \mathrm{~min}$. While ballistic movement is unlikely under these experimental conditions, this calculation underlines the high mobility of D. magna relative to smaller zooplankton species.

In addition to high feeding efficiency, it is also possible that other behavioral, physical, and chemical attributes of consumer-resource interactions contributed to the homogenization of algal distribution. For example, turbulence generated by copepods, krill, and some species of gelatinous zooplankton, has been suggested to be an important source of mixing in the ocean and lakes, perhaps even of similar importance as motion due to winds and tides (Huntley and Zhou 2004, Katija 2012). In our tanks, this effect could be even more important because of the relative small dimensions of the tank compared to real lakes and because $C$. vulgaris is a non-motile algae and cannot regulate its position in the water column.

It is also possible that Daphnia presence could affect growth rates of algae, either by altering rates of nutrient recycling or by simply triggering changes in morphology and life-history of algae (Long et al. 2007, Lass and Spaak 2003) via both consumptive and non-consumptive effects of Daphnia on algae (Scrimgeour et al. 1991). Lower growth rates could inhibit the formation of patchiness that we observed in the tanks without Daphnia.

Spatial self-organization has long been recognized as a critical process in consumer-resource systems. It can provide a stabilizing force, influencing the persistence of both consumer and resource populations and is accordingly a key factor determining food-web structure and biodiversity (de Roos et al. 1991, Hassell et al. 1991, van de Koppel et al. 2005). Our experiments demonstrate an equally important alternative effect: strong predation pressure combined with high mobility can homogenize the spatial distribution of resources. This scenario is similar to expansive consumer fronts seen in snails feeding on marine eelgrass beds, which can also homogenize spatial structure (Silliman et al. 2013). Thus, it remains an open question as to whether consumerresource interactions more commonly promote or inhibit spatial structure in their resources.

\section{ACKNOWLedGMents}

We thank O. Alexander, B. Liu, M. Phanouvong, A. Bowman, J. Roszell and S. Perim for their help with data collection. M. Cornish and M. Davies assisted with many technical aspects of the Limnotron. Financial support for this research was provided by a Discovery Grant to J. M. Fryxell from NSERC.

\section{Literature Cited}

Abraham, E. R. 1998. The generation of plankton patchiness by turbulent stirring. Nature 391:577-580.

Belsky, A. J. 1994. Influences of trees on savanna productivity: tests of shade, nutrients, and tree-grass competition. Ecology 75:922-932.

Betini, G. S., J. Roszell, A. Heyland, and J. M. Fryxell. 2016. Calcium interacts with temperature to influence Daphnia movement rates. Royal Society Open Science 3:160537.

Breheny, P., and W. Burchett. 2013. Visualization of regression models using visreg (). Technical report. University of Kentucky. https://CRAN.R-project.org/package=visreg

Brooks, J. L., and S. I. Dodson. 1965. Predation, body size, and composition of plankton. Science 150:28-35.

Burnham, K., D. Anderson, and K. Huyvaert. 2011. AIC model selection and multimodel inference in behavioral ecology: some background, observations, and comparisons. Behavioral Ecology and Sociobiology 65:23-35.

Cadotte, M. W., J. A. Drake, and T. Fukami. 2005. Constructing nature: laboratory models as necessary tools for investigating complex ecological communities. Advances in Ecological Research 37:333-353.

Dauta, A., J. Devaux, F. Piquemal, and L. Boumnich. 1990. Growth rate of four freshwater algae in relation to light and temperature. Hydrobiologia 207:221-226.

de Roos, A. M., E. Mccauley, and W. G. Wilson. 1991. Mobility versus density-limited predator-prey dynamics on different spatial scales. Proceedings of the Royal Society B 246: 117-122.

de Roos, A. M., E. McCauley, and W. G. Wilson. 1998. Pattern formation and the spatial scale of interaction between predators and their prey. Theoretical Population Biology 53:108-130.

Dobiesz, N. E., and N. P. Lester. 2009. Changes in mid-summer water temperature and clarity across the great lakes between 1968 and 2002. Journal of Great Lakes Research 35: 371-384.

Dodson, S. I., S. Ryan, R. Tollrian, and W. Lampert. 1997. Individual swimming behavior of Daphnia: effects of food, light and container size in four clones. Journal of Plankton Research 19:1537-1552.

Flaxman, S. M., and Y. Lou. 2009. Tracking prey or tracking the prey's resource? Mechanisms of movement and optimal habitat selection by predators. Journal of Theoretical Biology 256:187-200. 
Gurney, W. S. C., A. R. Veitch, I. Cruickshank, and G. McGeachin. 1998. Circles and spirals: population persistence in a spatially explicit predator-prey model. Ecology 79:2516-2530.

Hassell, M. P. 1984. Parasitism in patchy environments: inverse density dependence can be stabilizing. Mathematical Medicine and Biology 1:123-133.

Hassell, M. P., H. N. Comins, and R. M. Mayt. 1991. Spatial structure and chaos in insect population dynamics. Nature 353:255-258.

Hondzo, M., and H. G. Stefan. 1993. Regional water temperature characteristics of lakes subjected to climate change. Climatic Change 24:187-211.

Huntley, M., and M. Zhou. 2004. Influence of animals on turbulence in the sea. Marine Ecology Progress Series 273:65-79.

Jarvis, L., K. McCann, T. Tunney, G. Gellner, and J. M. Fryxell. 2016. Early warning signals detect critical impacts of experimental warming. Ecology and Evolution 6:6097-6106.

Katija, K. 2012. Biogenic inputs to ocean mixing. Journal of Experimental Biology 215:1040-1049.

Kilham, S. S., D. A. Kreeger, S. G. Lynn, C. E. Goulden, and L. Herrera. 1998. COMBO: a defined freshwater culture medium for algae and zooplankton. Hydrobiologia 377:147-159.

Klausmeier, C. A. 1999. Regular and irregular patterns in semiarid vegetation. Science 284:1826-1828.

Kondoh, M. 2007. Anti-predator defence and the complexitystability relationship of food webs. Proceedings of the Royal Society B 274:1617-1624.

Lampert, W. 2005. Vertical distribution of zooplankton: density dependence and evidence for an ideal free distribution with costs. BMC Biology 3:10.

Lampert, W., W. Fleckner, H. Rai, and B. E. Taylor. 1986. Phytoplankton control by grazing zooplankton: A study on the spring clear-water phase 1. Limnology and Oceanography 31:478-490.

Lampert, W., E. McCauley, and B. F. J. Manly. 2003. Trade-offs in the vertical distribution of zooplankton: ideal free distribution with costs? Proceedings of the Royal Society B 270:765-773.

Lass, S., and P. Spaak. 2003. Chemically induced anti-predator defences in plankton: a review. Hydrobiologia 491:221-239.

Levin, S. A., and L. A. Segel. 1976. Hypothesis for origin of planktonic patchiness. Nature 259:659-659.

Liu, Q.-X., A. Doelman, V. Rottschafer, M. de Jager, P. M. J. Herman, M. Rietkerk, and J. van de Koppel. 2013. Phase separation explains a new class of self-organized spatial patterns in ecological systems. Proceedings of the National Academy of Sciences USA 110:11905-11910.

Long, J. D., G. W. Smalley, T. Barsby, J. T. Anderson, and M. E. Hay. 2007. Chemical cues induce consumer-specific defenses in a bloom-forming marine phytoplankton. Proceedings of the National Academy of Sciences USA 104:10512-10517.

McCann, K. S., J. B. Rasmussen, and J. Umbanhowar. 2005. The dynamics of spatially coupled food webs: spatially coupled food webs. Ecology Letters 8:513-523.
McCauley, E., and W. W. Murdoch. 1987. Cyclic and stable populations: plankton as paradigm. American Naturalist 129:97-121.

McCauley, E., R. M. Nisbet, W. W. Murdoch, A. M. de Roos, and W. S. C. Gurney. 1999. Large-amplitude cycles of Daphnia and its algal prey in enriched environments. Nature 402:653-656.

Mougi, A., and M. Kondoh. 2012. Diversity of interaction types and ecological community stability. Science 337:349-351.

O'Keefe, T. C., M. C. Brewer, and S. I. Dodson. 1998. Swimming behavior of Daphnia: its role in determining predation risk. Journal of Plankton Research 20:973-984.

Pinheiro, J. C., and D. M. Bates. 2009. Mixed-effects models in S and S-PLUS. Springer, New York, New York, USA.

Pinheiro, J. C., D. M. Bates, S. DebRoy, and D. Sarkar. 2016. nlme: linear and nonlinear mixed effects models. R package version 3.1-123. http://CRAN.R-project.org/package=nlme.

R Core Team. 2015. R: A language and environment for statistical computing. R Foundation for Statistical Computing, Vienna, Austria. www.r-project.org

Resetarits, W. J., and J. Bernardo. 1998. Experimental ecology: issues and perspectives. Oxford University Press, New York, New York, USA.

Rietkerk, M., and J. van de Koppel. 2008. Regular pattern formation in real ecosystems. Trends in Ecology and Evolution 23:169-175.

Scheffer, M. 2004. Ecology of Shallow Lakes. Springer Science \& Business Media, New York, New York, USA.

Schindler, D. W. 1998. Whole-ecosystem experiments: replication versus realism: the need for ecosystem-scale experiments. Ecosystems 1:323-334.

Scrimgeour, G. J., J. M. Culp, M. L. Bothwell, F. J. Wrona, and M. H. McKee. 1991. Mechanisms of algal patch depletion: importance of consumptive and non-consumptive losses in mayfly-diatom systems. Oecologia 85:343-348.

Selander, E., J. Kubanek, M. Hamberg, M. X. Andersson, G. Cervin, and H. Pavia. 2015. Predator lipids induce paralytic shellfish toxins in bloom-forming algae. Proceedings of the National Academy of Sciences USA 112:6395-6400.

Silliman, B. R., M. W. McCoy, C. Angelini, R. D. Holt, J. N. Griffin, and J. van de Koppel. 2013. Consumer fronts, global change, and runaway collapse in ecosystems. Annual Review of Ecology, Evolution, and Systematics 44:503-538.

Solé, R. V., and J. Bascompte. 2012. Self-organization in complex ecosystems. Princeton University Press, Princeton, New Jersey, USA.

van de Koppel, J., M. Rietkerk, N. Dankers, P. M. J. Herman, E. J. B. Losos, and A. E. J. P. Grover. 2005. Scale-dependent feedback and regular spatial patterns in young mussel beds. American Naturalist 165:E66-E77.

Zuur, A., E. N. Ieno, N. Walker, A. A. Saveliev, and G. M. Smith. 2009. Mixed effects models and extensions in ecology with R. Springer Science \& Business Media, New York, New York, USA. 